\title{
Оценка термодинамической эффективности работы твердотельных охладителей и генераторов на мультикалорическом эффекте
}

\author{
(c) А.С. Старков, ${ }^{1}$ О.В. Пахомов, ${ }^{1}$ В.В. Родионов, ${ }^{2}$ A.А. Амиров, ${ }^{2,3}$ И.А. Старков ${ }^{1,4}$ \\ ${ }^{1}$ Университет ИТМО, \\ 197101 Санкт-Петербург, Россия \\ 2 Лаборатория новых магнитных материалов \& Институт фризико-математических наук и информационных технологий, \\ Балтийский федеральный университет им. И. Канта, \\ 236041 Калининград, Россия \\ ${ }^{3}$ Институт фризики им. Х.И. Амирханова ДагНЦ РАН, \\ 367003 Махачкала, Россия \\ ${ }^{4}$ Санкт-Петербургский национальный исследовательский академический университет РАН, \\ 194021 Санкт-Петербург, Россия \\ e-mail: ferroelectrics@ya.ru
}

(Поступило в Редакцию 18 января 2018 г.)

Исследована эффективность использования мультикалорического эффекта $(\mu \mathrm{CE})$ в твердотельных охлаждающих системах и произведено сравнение с одиночными калорическими эффектами. Предложенный подход проиллюстрирован на примере цикла Брайтона для $\mu \mathrm{CE}$ и магнитокалорического эффекта. На основе проведенных экспериментов для двухслойного композита $\mathrm{Fe}_{48} \mathrm{Rh}_{52}-\mathrm{PbZr}_{0.53} \mathrm{Ti}_{0.47} \mathrm{O}_{3}$ построена зависимость относительной эффективности от температуры и дана оценка температурного диапазона, где $\mu \mathrm{CE}$ имеет преимущество над магнитокалорическим эффектом. Произведено сравнение разработанной теории мультикалорического эффекта с полученными экспериментальными данными.

DOI: $10.21883 /$ JTF.2019.04.47318.34-18

\section{Введение}

При анализе эффективности термодинамических циклов тепловых машин используются различные коэффициенты. Для прямых циклов, применяющихся в генераторах, - это коэффициент полезного действия (КПД) $\xi$, определяемый для теплосиловых циклов как отношение полученной работы к подведенной теплоте. В случае обратных циклов, служащих основой работы холодильных машин и установок, - это холодильный (СОР coefficient of perfomance) и отопительный (HF - heating factor) коэффициенты. Параметр СОР определяется как отношение количества теплоты, отнятой от охлаждаемого объекта (холодопроизводительности), к работе, затраченной на организацию цикла. Отопительный коэффициент используется для оценки эффективности тепловых насосов и равен отношению количества тепла, сообщенного нагреваемому объекту, к величине работы. Для прямых циклов тепловой КПД - это отношение теплоты, отдаваемой горячему объекту, к работе, затраченной на осуществление цикла [1]. Отметим, что все эти коэффициенты разделяются на теоретические и действительные. Теоретические коэффициенты характеризуют эффективность идеальных термодинамических циклов, а не реальных устройств, работающих по данным циклам. Действительные коэффициенты определяются из результатов экспериментального исследования реальных установок и учитывают тепловые потери и дополнительные затраты энергии.
Величина термического КПД для всех устройств меньше единицы, а для холодильного и отопительного коэффициентов теория допускает существование сколь угодно больших значений при малых разностях температур в цикле. Величина теоретического холодильного коэффициента СОР меняется для парокомпрессионных холодильников в пределах от 4 до 7 [1]. Ввиду этого величина СОР является не вполне объективной, и для оценки эффективности какого-либо цикла $L$ обычно используют относительные коэффициенты $\eta=\left(\mathrm{COP}_{L} / \mathrm{COP}_{\text {Carnot }}\right) 100 \%$ и $\kappa=\xi_{L} / \xi_{\text {Carnot }} 100 \%$, где $\mathrm{COP}_{\text {Carnot }}$ и $\xi_{\text {Carnot }}$ - холодильный коэффициент и КПД цикла Карно. Так как цикл Карно является наиболее эффективным среди всех термодинамических циклов, то его эффективность принимается за единицу. Для парокомпрессионных холодильников и воздушных холодильных машин $\eta$ обычно не превышает $20 \%$, причем дальнейшее увеличение эффективности затруднено в силу ряда объективных причин [1]. Следовательно, поиск новых принципов работы тепловых машин представляется одной из наиболее важных задач современной науки и техники.

Среди новых технологий преобразования тепловой энергии наиболее перспективной, на наш взгляд, является твердотельное охлаждение и генерация энергии. Основное преимущество твердотельного охлаждения заключается в значительно большей плотности твердого тела по сравнению с плотностью пара и газа в парокомпрессионных устройствах. Вследствие этого изменение энтропии на единицу объема в твердых материалах 
в 6-8 раз выше, чем в газе [2], что позволяет создавать высокоэффективные устройства для охлаждения и генерации энергии. В дальнейшем основное внимание уделим твердотельным охладителям, так как в настоящее время они, с нашей точки зрения, более конкурентоспособны. У них нет движущихся частей, поэтому твердотельные охладители более компактны, надежны и практически бесшумны. Более того, используемые в них материалы являются экологически безопасными в отличие от множества хладагентов, применяющихся в настоящее время в холодильной технике. Современные твердотельные охладители разделяется на два типа: термоэлектрические [1] и на калорических эффектах [3]. Так как эффект Пельтье, лежащий в основе работы термоэлектрического охладителя, является термодинамически необратимым, то его эффективность является малой и составляет не более 7\% от эффективности цикла Карно. Гораздо более эффективным является охлаждение, основанное на калорических эффектах [3-6]. Для рабочих тел, обладающих магнитокалорическим эффектом (МСЕ), эффективность составляет порядка 70\% [7]. Для устройств на электрокалорическом эффекте (ЕСЕ) эта эффективность оценивалась в 60\% [2]. Эффективность эластокалорических циклов может достигать 84\% [8]. Необходимо подчеркнуть, что эффективность работающих прототипов несколько меньше. Так, согласно [9], действительная эффективность для ЕСЕ составляет $31 \%$, что существенно превосходит соответствующие значения для холодильных машин (20\% [1]). Примерно такой же эффективностью обладал один из первых прототипов электрокалорического охладителя [10], для которого эксергетическая эффективность (несколько отличающаяся от $\eta$ ) равнялась $58 \%$. Наивысшей экспериментально подтвержденной эффективностью на данный момент обладают магнитоэлектрические материалы (до 60\% [11]).

Однако, несмотря на высокую эффективность, технология охлаждения, основанная на калорических эффектах, широкого распространения не получила из-за высокой стоимости материалов и отсутствия надежных тепловых ключей. Ввиду этого было предложено использовать не одиночный калорический эффект, а согласованное действие нескольких физических полей, приводящих к появлению мультикалорического эффекта ( $\mu \mathrm{CE})$ [6,12-14]. Твердотельные охладители на $\mu \mathrm{CE}$ обладают не только рядом технических преимуществ, но и позволяют увеличить эффективность подобных устройств. Детально этот вопрос ранее не рассматривался. Его исследованию и посвящена настоящая работа.

\section{1. Основы термодинамики мультикалорического эффекта}

Термодинамика мультикалорического эффекта неоднократно рассматривалась в литературе (см., например, $[3,4,6,14])$. К сожалению, в настоящее время име- ется множество работ, содержащих грубые ошибки при описании теории мультикалорического эффекта. Часть из этих ошибок разобрана в $[15,16]$. Ввиду этого мы кратко изложим основные положения термодинамики мультикалориков. Для описания термодинамической системы обычно используют внутреннюю энергию $U$ и свободную энергию Гиббса $F$, связанные между собой соотношением $[3,4,14]$ :

$$
F=U-T S-x_{i} X_{i}
$$

Здесь $T-$ температура, $S-$ энтропия, $x_{i}-$ обобщенные силы, $X_{i}$ - обобщенные координаты $(i=1,2, \ldots, n)$. При записи уравнения (1) использовалось соглашение Эйнштейна: по повторяющимся индексам производится суммирование. Будем предполагать, что $U$ зависит только от обобщенных сил и координат, но не зависит от их производных. Свободная энергия, в которой имеется зависимость от производных, рассмотрена в [4]. Условие экстремальности $F$ вида (1) ведет к соотношением

$$
X_{i}=\frac{\partial U}{\partial x_{i}}, \quad S=-\frac{\partial F}{\partial T}
$$

Дифференциал энтропии с учетом соотношений Максвелла $\partial S / \partial x_{i}=\partial X_{i} / \partial T$ и определения теплоемкости $C=T \partial S / \partial T$ при постоянных внешних полях записывается в виде $[3,4]$

$$
d S=\frac{C}{T} d T+\frac{\partial X_{i}}{\partial T} d x_{i}
$$

Отсюда получаем уравнения основных термодинамических процессов

$$
d S_{\mathrm{ist}}=\frac{\partial X_{i}}{\partial T} d x_{i}, \quad d T_{\mathrm{ad}}=-\frac{T}{C} \frac{\partial X_{i}}{\partial T} d x_{i} .
$$

Первое уравнение в (4) описывает изотермическое изменение энтропии, второе - адиабатическое изменение температуры. Оба вышеуказанных процесса являются частью различных термодинамических циклов. Например, цикл Карно состоит из двух изотерм и двух адиабат. Еще одним важным процессом является изополевой (isofield), когда внешние силы остаются неизменными. Для этого цикла связь изменений энтропии и температуры находится из равенства (3), в котором нужно положить $d x_{i}=0$. Коэффициенты перед $d x_{i}$ в первом и втором уравнениях (4) называются соответственно пиро- и калорическими коэффициентами. Наиболее известными являются пироэлектрический, пиромагнитный, электрокалорический и магнитокалорический коэффициенты $[3,4]$.

Отметим принципиальную разницу между двумя уравнениями в (4) в неодномерной $(n>1)$ термодинамике. Дифференциал энтропии является полным. Значит, изменение энтропии в произвольном процессе описывается криволинейным интегралом от начальной до конечной точек процесса, причем значение интеграла не зависит 
от пути интегрирования. Второе уравнение в (4) следует рассматривать как дифференциальное. Если обобщенная сила одна $(n=1)$, то изменение температуры определяется только значениями этой силы в начальной и конечной точках. В общем случае $(n>1)$ изменение температуры зависит не только от начальных и конечных значений обобщенных сил, но и от последовательности их применения. Следовательно, одной из важных задач, которые необходимо будет рассмотреть в многомерной термодинамике, является задача о поиске соотношения между $x_{i}$, которое обеспечивает наибольшее изменение температуры.

В большинстве случаев можно ограничиться линейной зависимостью между обобщенными силами и обобщенными координатами. При этом предположении внутренняя энергия будет квадратичной формой от обобщенных координат $U=a_{i j} X_{i} X_{j} / 2$, где $a_{i j}=a_{j i}-$ некоторые коэффициенты, которые могут зависеть от температуры. Связь между обобщенными силами и обобщенными координатами может быть записана в виде $x_{i}=a_{i j} X_{j}$ или

$$
X_{i}=\chi_{i j} x_{j}
$$

где матрица обобщенных восприимчивостей $\chi$ является обратной к матрице $a$. Для линейной зависимости (5) равенства (4) принимают вид

$$
d S_{\text {ist }}=\frac{\partial \chi_{i j}}{\partial T} x_{i} d x_{j}, \quad d T_{\mathrm{ad}}=-\frac{T}{C} \frac{\partial \chi_{i j}}{\partial T} x_{i} d x_{j} .
$$

Отсюда следует, что в $\mu$ СЕ вносит вклад любой элемент матрицы $\chi$, зависящий от температуры, и этот вклад пропорционален производной $\partial \chi_{i j} / \partial T$.

В качестве примера расчета эффективности термодинамического цикла для мультикалорика рассмотрим линейный сегнетомагнетик, свободная энергия которого $F$ имеет вид

$$
F=\frac{P^{2}}{2 \varepsilon_{0} \chi_{e}}+\frac{M^{2}}{2 \mu_{0} \chi_{m}}+\alpha M P-E P-M H .
$$

Здесь $P-$ поляризация, $M-$ намагниченность, $E$ и $H$ - напряженности электрического и магнитного полей, $\varepsilon_{0}$ и $\mu_{0}$ - электрическая и магнитная постоянные, $\alpha-$ магнитоэлектрический (ME) коэффициент. Коэффициенты $\alpha, \chi_{e}, \chi_{m}$ предполагаются не зависящими от полей $E, H$, но зависят от температуры $T$. Именно температурная зависимость этих коэффициентов и обусловливает наличие калорических эффектов.

Напомним, что сегнетомагнетики - вещества, обладающие одновременно магнитным и электрическим упорядочением $[17,18]$. Взаимосвязь магнитного и электрического поля приводит к появлению магнитоэлектрического эффекта. При приложении к таким веществам внешнего электрического поля происходит изменение намагниченности, и, наоборот, при приложении внешнего магнитного поля происходит изменение поляризации. Уравнения, описывающие зависимость электрического и магнитного полей, являются частным случаем уравнений (2), и в случае свободной энергии (7) имеют вид [17]

$$
E=\frac{P}{\varepsilon_{0} \chi_{e}}+\alpha M, \quad H=\frac{M}{\mu_{0} \chi_{m}}+\alpha P .
$$

Следует подчеркнуть, что результат подстановки (8) в (7) может быть записан в виде $F=-(P E+M H) / 2$, т.е. так же, как и в обычной электро- и магнитостатике при отсутствии магнитоэлектрического взаимодействия [19].

В естественных сегнетомагнетиках величина MEэффекта мала $\left(\alpha^{2} \varepsilon_{0} \mu_{0} \chi_{e} \chi_{m} \ll 1\right)$ и, кроме того, в большинстве материалов он наблюдается при температурах, значительно ниже комнатной [18]. Композиционные мультиферроики представляют собой двухфазные структуры, состоящие из связанных между собой магнитострикционной и пьезоэлектрической подсистем. Величина ME-эффекта в таких структурах на несколько порядков превышает величину МЕ-эффекта в естественных сегнетомагнетиках.

Введем еще один магнитоэлектрический коэффициент $\beta=\alpha \varepsilon_{0} \mu_{0} \chi_{e} \chi_{m}$ и, используя малость $\beta$, перепишем равенство (8) в виде

$$
P=\varepsilon_{0} \chi_{e} E-\beta H, \quad M=-\beta E+\mu_{0} \chi_{m} H .
$$

Отсюда видно, что диагональные элементы матрицы $\chi$ размером $2 \times 2$ в этом случае равны электрической и магнитной восприимчивостям, а внедиагональные равны МЕ-коэффициенту $\beta$. Коэффициент $\beta$ называется МЕвосприимчивостью.

Выписанные соотношения позволяют найти тепловые характеристики сегнетомагнетика, необходимые для описания термодинамических циклов. Наиболее просто находится энтропия $S$

$$
S=-\frac{\partial F}{\partial T}=\frac{P^{2}}{2 \varepsilon_{0} \chi_{e}^{2}} \frac{\partial \chi_{e}}{\partial T}+\frac{M^{2}}{2 \mu_{0} \chi_{m}^{2}} \frac{\partial \chi_{m}}{\partial T}-M P \frac{\partial \alpha}{\partial T} .
$$

Если использовать соотношения (9), то (10) можем переписать через независимые переменные $E$ и $H$

$$
S=\frac{\varepsilon_{0} E^{2}}{2} \frac{\partial \chi_{e}}{\partial T}+\frac{\mu_{0} H^{2}}{2} \frac{\partial \chi_{m}}{\partial T}-E H \frac{\partial \beta}{\partial T} .
$$

Из (11) следует, что изменение энтропии при изотермическом процессе состоит из трех слагаемых, каждое из которых отвечает соответствующему калорическому эффекту. Первое задает ЕСЕ, второе - магнитоэлектрокалорический эффект (MECE), третье - MCE. Теплоемкость $C$ обычным образом находится из энтропии и представляется в виде суммы двух слагаемых

$$
C=C_{0}+\Delta C, \quad \Delta C=T \frac{\partial S}{\partial T},
$$

где $C_{0}-$ теплоемкость в отсутствие поля. В теории Ландау $C_{0}$ считается линейной функцией абсолютной температуры [20], но для комнатных температур и 
малом изменении температуры (меньше одного градуса) в цикле можно считать $C_{0}$ постоянной. Из трех слагаемых, входящих в $\Delta C$, выпишем только одно, которое обусловлено магнитоэлектрическим эффектом $\Delta C_{E H}$ :

$$
\Delta C_{E H}=-T \frac{\partial^{2} \beta}{\partial T^{2}} E H
$$

Эта часть теплоемкости линейна как по электрическому, так и по магнитному полю. Кроме того, при изменении направления одного из полей она меняет знак. Пироэлектрический и пиромагнитный коэффициенты имеют вид

$$
\frac{\partial P}{\partial T}=\varepsilon_{0} \frac{\partial \chi_{e}}{\partial T} E-\frac{\partial \beta}{\partial T} H, \quad \frac{\partial M}{\partial T}=-\frac{\partial \beta}{\partial T} E+\mu_{0} \frac{\partial \chi_{m}}{\partial T} .
$$

Полученные формулы (11)-(14) позволяют выписать уравнения основных термодинамических процессов для сегнетомагнетика

$$
\begin{gathered}
d S=\frac{C}{T} d T+\frac{\partial P}{\partial T} d E+\frac{\partial M}{\partial T} d H \\
d T_{a d}=-\frac{T}{C}\left(\varepsilon_{0} \frac{\partial \chi_{e}}{\partial T} E d E+\mu_{0} \frac{\partial \chi_{m}}{\partial T} H d H-\frac{\partial \beta}{\partial T} d(E H)\right) .
\end{gathered}
$$

Из (15) видно, что МЕСЕ пропорционален изменению как электрического, так и магнитного полей. Коэффициент пропорциональности естественно назвать магнитоэлектрокалорическим коэффициентом, $\gamma_{E H}=T / C \partial \beta / \partial T$.

В рассматриваемой модели изменение температуры в адиабатическом процессе определяется только начальными и конечными значениями электрического и магнитного полей $E, H$. Это свойство является следствием предположения об отсутствии зависимости материальных постоянных от значений внешних полей. Напомним, что для изменения энтропии это утверждение справедливо при любых зависимостях $\chi_{e}, \chi_{m}$ от $E, H$.

\section{2. Цикл Брайтона и его холодильный коэффициент}

Одной из основных характеристик эффективности холодильной установки является безразмерные холодильный и отопительный коэффициенты - COP и НF. Они определяются соответственно как отношение количества теплоты $Q_{C}$, отнятой от охлаждаемого объекта (холодопроизводительности), к работе $W$, затраченной на организацию цикла, $\mathrm{COP}=Q_{c} / W$, и как отношение количества тепла, сообщенной нагреваемому объекту, к величине работы $\mathrm{HF}=Q_{h} / W$. Для произвольного термодинамического цикла, описываемого в системе координат $(S, T)$ замкнутой кривой $L$, которая состоит из верхней $L_{h}$ (горячей) и нижней $L_{c}$ (холодной) частей, $L=L_{c}+L_{h}$ (рис. 1), СОР и НF выражаются следующим

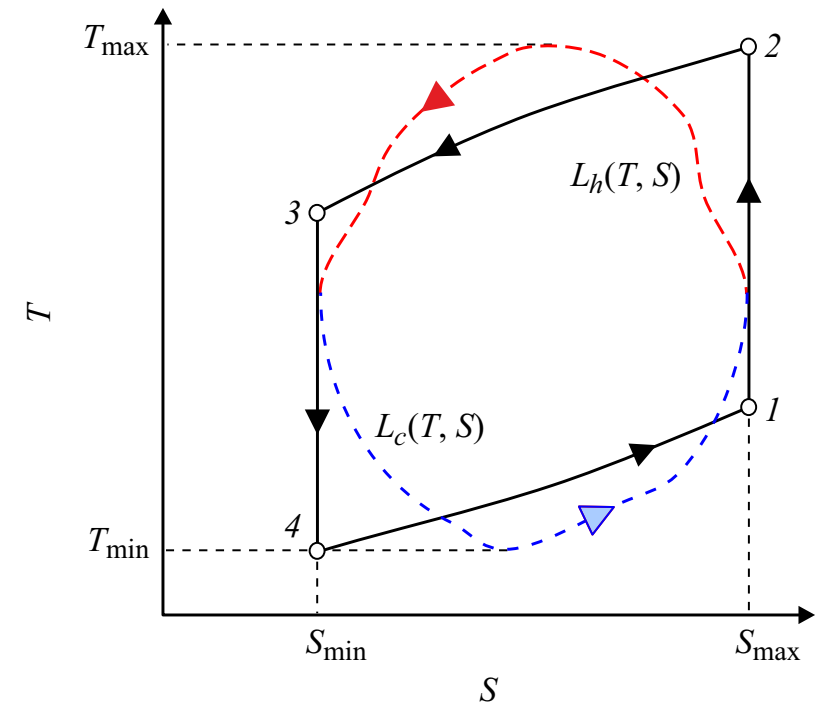

Рис. 1. Общий термодинамический цикл (штриховые линии) и цикл Брайтона (непрерывная линия).

образом:

$$
\mathrm{COP}=-\frac{\int_{L_{c}} T d S}{\oint_{L} T d S}, \quad \mathrm{HF}=\frac{\int_{L_{h}} T d S}{\oint_{L} T d S} .
$$

Например, для цикла Карно кривая $L$ является прямоугольником, для цикла Брайтона - криволинейной трапецией (рис. 1). Интегрирование в криволинейном интеграле, как и для всех холодильных машин, осуществляется против часовой стрелки. Эффективность генераторов характеризуется через КПД цикла $\xi$, определяемом как отношение полученной работы к подведенной теплоте $\xi=\oint_{L} T d S / \int_{L_{c}} T d S$. Здесь интегрирование осуществляется по часовой стрелке.

Применим выведенные соотношения для нахождения эффективности цикла Брайтона, который часто встречается как в парокомпрессионных холодильниках [1], так и в холодильниках на MCE [21]. Будем рассматривать этот цикл для рабочего тела, обладающего $\mu \mathrm{CE}$. Цикл Брайтона состоит из двух адиабат 1-2 и 3-4 и двух изополевых кривых 2-3, 4-1 (рис. 1). В дальнейшем значения физических величин на кривых и в четырех угловых точках цикла будет снабжать соответствующими индексами. На участке $1-2$ происходит адиабатическое приложение внешних полей $(E$ и $H)$, которые меняются от значений $E_{1}, H_{1}$ до $E_{2}, H_{2}$. Температура рабочего тела (РТ) при этом возрастает от $T_{1}$ до $T_{2}$. На участке $2-3$ тепло от РТ передается в окружающую среду, и РТ остывает до температуры $T_{3}$. На участке $3-4$ внешние поля адиабатически снимаются, что приводит к уменьшению температуры РТ до значения $T_{4}$. На последней части цикла $1-4$ РТ принимает теплоту от охлаждаемого объекта, и его температура поднимается до первоначальной $T_{1}$.

Изменения температуры $T_{2}-T_{1}, T_{3}-T_{4}$ могут быть рассчитаны по формуле (7). На оставшихся участ- 
ках 2-3,4-1 внешние поля постоянны, и изменение температуры мало. Следовательно, теплоемкость на этих отрезках можно считать постоянной, а зависимость $T(S)$ - линейной, например, $T=T_{4}+\left(S-S_{4}\right) / C_{41}$. Тогда интегралы в определении СОР (16) можно заменить на площади соответствующих трапеций, и в результате получаем приближенную формулу для вычисления СОР

$$
\mathrm{COP}=\frac{T_{1}+T_{4}}{T_{2}+T_{3}-T_{1}-T_{4}} .
$$

Аналогичные формулы могут быть выписаны для КПД и отопительного коэффициента.

По известным значениям СОР (16) и $\mathrm{COP}_{\text {Carnot }}$ легко находится относительная эффективность $\eta=\left(\mathrm{COP} / \mathrm{COP}_{\text {Carnot }}\right) 100 \%$. Величины, входящие в формулу (17), могут быть получены либо в рамках какой-либо теоретической модели (например, модели Гинзбурга-Ландау), либо на основе экспериментальных данных.

Для сравнения эффективности различных циклов естественно потребовать, чтобы для них адиабатическое изменение температуры $T_{2}-T_{1}$ было одним и тем же. Тогда СОР определяется в основном значением наклона линий 2, 3 и 4-1. А этот наклон обратно пропорционален изополевой теплоемкости $C$, которая определяется формулами (11), (12). Следовательно, наличие не одного, а нескольких полей приводит к более заметному изменению теплоемкости. Повышение же теплоемкости приводит к увеличению эффективности.

\section{3. Экспериментальное наблюдение калорических эффектов в композите}

Так как естественные сегнетомагнетики обладают малым магнитоэлектрическим коэффициентом, то был изготовлен двухслойный магнитоэлектрический сэндвич FeRh-PZT, состоящий из магнитного слоя железородиевого сплава $\mathrm{Fe}_{48} \mathrm{Rh}_{52}(\mathrm{FeRh})$ и пьезоэлектрического слоя $\mathrm{PbZ}_{0.53} \mathrm{Ti}_{0.47} \mathrm{O}_{3}$ (PZT) с толщиной $0.2 \mathrm{~mm}$ каждый. Выбор указанных соединений в качестве компонентов обоснован тем, что сплав $\mathrm{Fe}_{48} \mathrm{Rh}_{5} 2$ является материалом, в котором был обнаружен один из рекордных значений магнитокалорического эффекта [22], а соединение цирконата-титаната свинца (РZT) является одним из лучших и доступных пьезоэлектрических материалов. Сплавы Fe-Rh характеризуются тем, что демонстрирует гигантский отрицательный магнитокалорический эффект в области 300-350 K с метамагнитным переходом первого рода из антиферромагнитного состояния в ферромагнитное, сопровождающийся изменениями структуры $[23,24]$. Компоненты магнитоэлектрического композита предварительно шлифовались, их поверхности очищались в ультразвуковой ванне и склеивались с помощью специального эпоксидного клея. Контакты для приложения электрического поля изготавливались из серебряной пасты путем нанесения на плоскости пьезоэлектрического слоя с обеих сторон. Были проведены исследования магнитных, магнитокалорических и магнитоэлектрических свойств композита FeRh-PZT в области комнатной температуры. Магнитные свойства композита изучались на вибрационном магнитометре LakeShore7400. Намагниченность была рассчитана как суммарный магнитный момент на единицу массы магнитной компоненты композита. Магнитокалорический эффект измерялся прямым методом, используя оригинальную и апробированную методику в переменном магнитном поле низкой частоты $[25,26]$. В измерениях магнитокалорического эффекта для регистрации адиабатического изменения температуры использовалась хромель-константановая дифференциальная микротермопара с диаметром проводов $25 \mu \mathrm{m}$, которая была склеена между пьезоэлектрическим и магнитным слоями. Для улучшения теплового контакта и уменьшения инерционности термопары концы термопары были сплющены до $3 \mu \mathrm{m}$ и сварены точечной микросваркой. Сигнал от термопары предварительно усиливался и измерялся с помощью селективного нановольтметра SR830. Для генерации переменного магнитного поля использовалась специально сконструированная система из четырех вращающихся постоянных магнитов амплитудой магнитного поля 0.62 Т и частотой до $25 \mathrm{~Hz}$ [26]. Магнитокалорический эффект в образце был исследован в двух режимах: с поданным на пьезоэлектрический слой композита постоянным электрическим напряжением и в случае, когда напряжение было отключено. Магнитоэлектрический эффект определялся через величину напряжения, возникаемого на концах образца при помещении его в переменное магнитное поле [27]. МЕ-сигнал измерялся с помощью селективного нановольтметра SR830, MEнапряжение было направлено параллельно магнитному полю. При экспериментальном наблюдении прямого МЕ-эффекта проще всего находить МЕ-коэффициент по напряжению $\alpha_{E}$, который определяется из выражения:

$$
\alpha_{E}=\frac{d E}{d H}=\frac{d V}{b d H}=\frac{V_{a c}}{b H_{a c}},
$$

где $V_{a c}$ - переменное напряжение, индуцируемое в PZTслое, $b$ - толщина пьезоэлектрического слоя, $H_{a c}-$ амплитуда переменного модулирующего магнитного поля. Нетрудно проверить, что МЕ-коэффициент по напряжению $\alpha_{E}$ связан с МЕ-коэффициентом $\alpha$ соотношением $\alpha=-\varepsilon \alpha_{E}$. При проведении экспериментов амплитуда переменного магнитного поля $H_{a c}$ составила $0.62 \mathrm{~T}$, а частота $-3 \mathrm{~Hz}$. Дополнительное постоянное магнитное поле в МЕ-измерениях не использовалось. Для описанного композита была исследована температурная зависимость изменения температуры $\Delta T_{\mathrm{MCE}}$ при МСЕ для магнитного поля $0.62 \mathrm{~T}$. Затем на образец было подано электрическое поле $25 \mathrm{~V}$ и было измерено изменение температуры $\Delta T_{\mu C E}$ при мультикалорическом эффекте (при одновременной подаче электрического и 


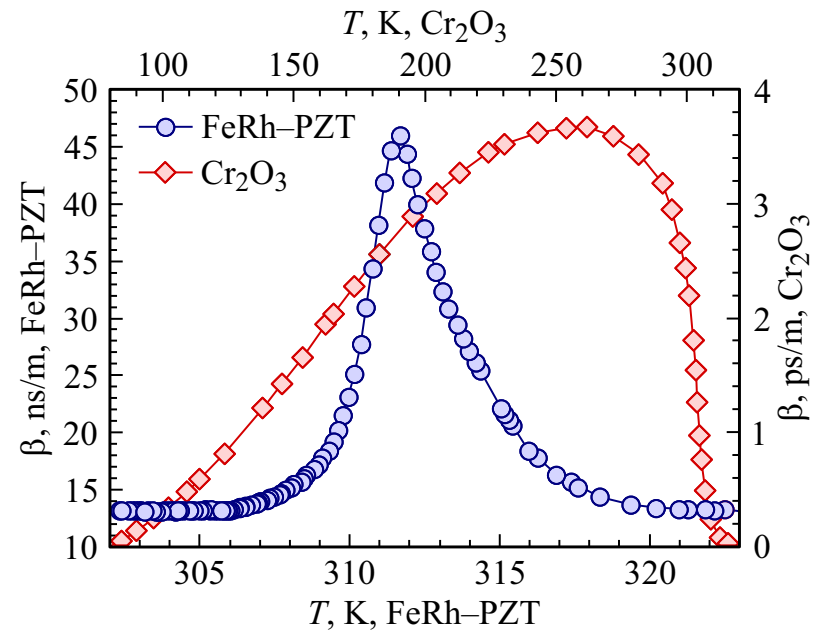

Рис. 2. Температурная зависимость магнитоэлектрической восприимчивости для $\mathrm{FeRh}-\mathrm{PZT}$ и $\mathrm{Cr}_{2} \mathrm{O}_{3}$.

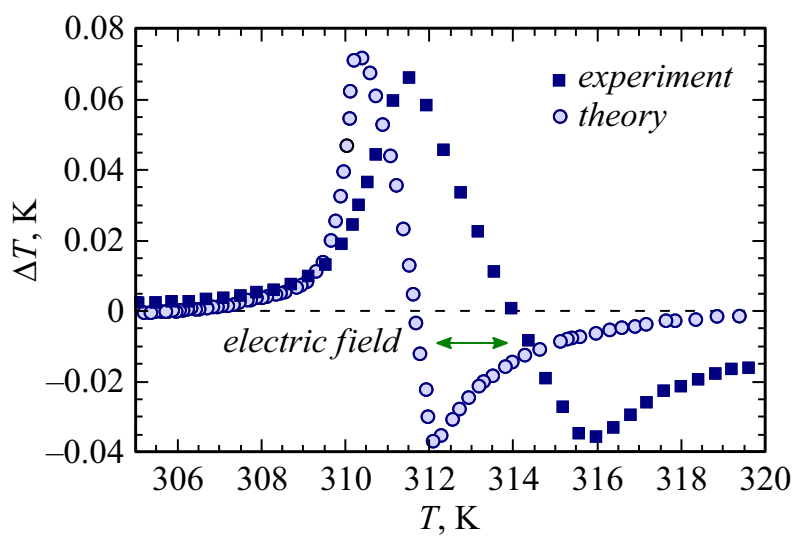

Рис. 3. Зависимость изменения температуры при магнитоэлектрокалорическом эффекте для FeRh-PZT.

магнитного полей). Параллельно с этим были произведены измерения зависимости магнитоэлектрического коэффициента от температуры. На основе произведенных измерений была построена зависимость магнитоэлектрической восприимчивости $\beta$ от температуры (рис. 2). На этом и последующих графиках (за исключением рис. 3) для сравнения приведены также данные для природного сегнетомагнетика $\mathrm{Cr}_{2} \mathrm{O}_{3}$, рассчитанные по данным работы [28]. Величина $\beta$ получилась на $2-3$ порядка меньше, чем в аналогичном композите $\mathrm{FeRH}_{-} \mathrm{BaTiO}_{3}$ [29]. По всей видимости, это вызвано существенно бо́льшими полями $\left(E \sim 2 \cdot 10^{8} \mathrm{~V} / \mathrm{m}\right)$ и малой толщиной слоев (толщина слоя FeRh составила $22 \mathrm{~nm})$.

В результате обработки экспериментальных данных по формуле $\Delta T_{\mathrm{MECE}}=\Delta T_{\mu \mathrm{CE}}-\Delta T_{\mathrm{MCE}}$ было получено, что величина MECE c высокой степенью точности совпадает с теоретической величиной, вычисленной по формуле (15) (рис. 3). Это подтверждает правильность выбранной модели (7), (15) для описания $\mu \mathrm{CE}$. Имеющиеся отличия в графиках вызваны тем обстоятель- ством, что магнитоэлектрическая восприимчивость, на основе которой рассчитывалась теоретическая кривая MECE, измерялась в отсутствие электрического поля. А в формуле (15) фигурирует эта же величина, но при наличии электрического поля, которое приводит к сдвигу температурной зависимости $\beta$ в сторону более высоких температур. При расчетах мы пренебрегали ECE, так как фазовый переход в PZT происходит вдали от комнатных температур, а в рассматриваемом температурном диапазоне свойства PZT можно считать не зависящими от $T$.

\section{4. Обсуждения и результаты}

На основе полученных результатов был построен графики зависимости магнитоэлектрокалорических коэффициентов $\gamma_{E H}$ для FeRh-PZT и $\mathrm{Cr}_{2} \mathrm{O}_{3}$ (рис. 4). Эти графики пропорциональны первой производной $\beta$ по температуре. Вторая производная определяет добавоч-

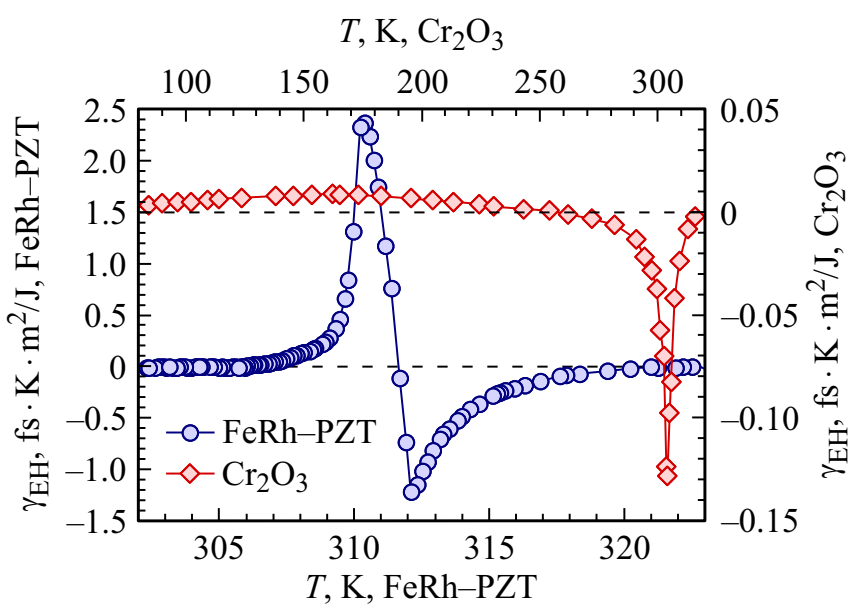

Рис. 4. Температурная зависимость магнитоэлектрокалорического коэффициента для $\mathrm{FeRh}-\mathrm{PZT}$ и $\mathrm{Cr}_{2} \mathrm{O}_{3}$.

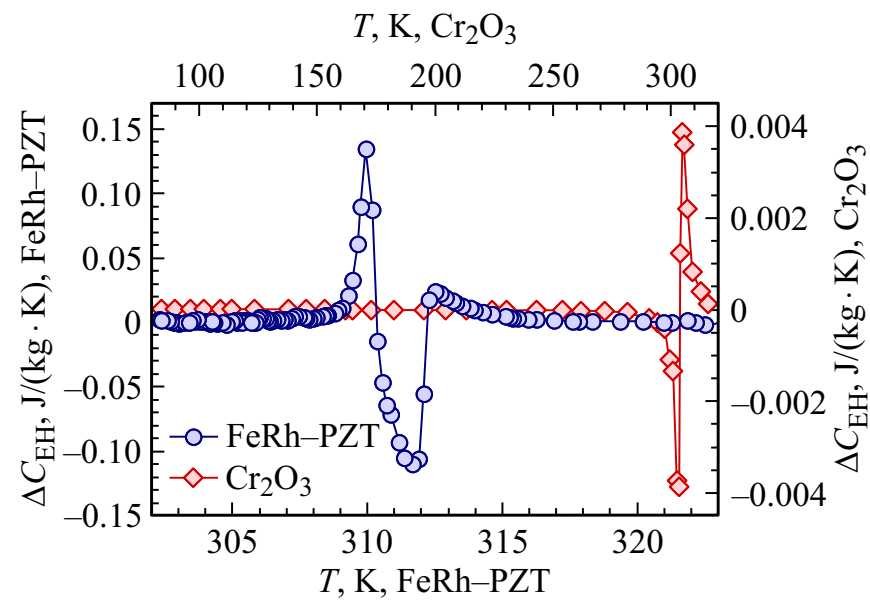

Рис. 5. Зависимость добавочной теплоемкости, обусловленной магнитоэлектрическим эффектом от температуры для $\mathrm{FeRh}-\mathrm{PZT}$ и $\mathrm{Cr}_{2} \mathrm{O}_{3}$. 


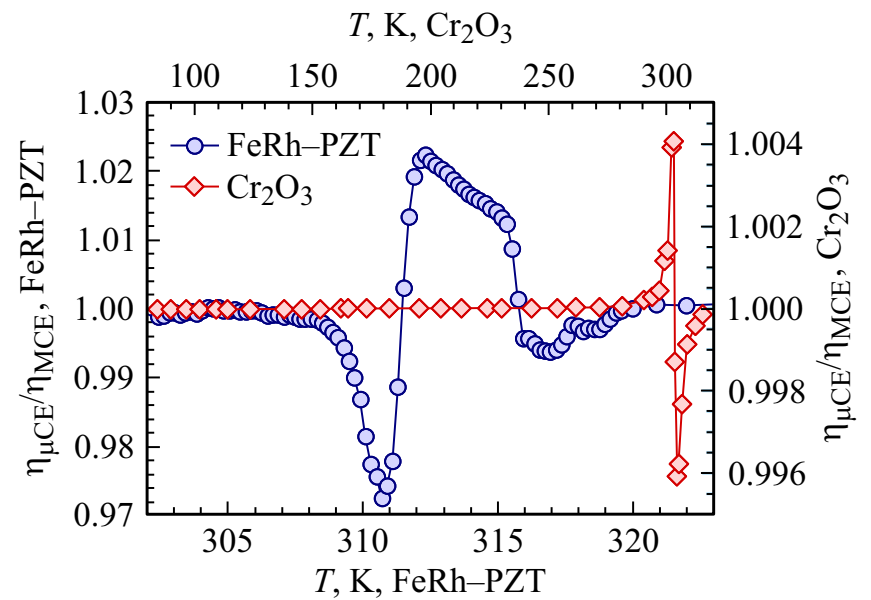

Рис. 6. Отношение эффективностей мультикалорического и магнитокалорического эффектов как функция температуры для FeRh-PZT и $\mathrm{Cr}_{2} \mathrm{O}_{3}$.

ный вклад в объемную теплоемкость, которая изображена на рис. 5. Вычисления для $\mathrm{Cr}_{2} \mathrm{O}_{3}$ проводились для электрического поля $4.8 \cdot 10^{7} \mathrm{~V} / \mathrm{m}$ и магнитного поля в 4T. Также эта производная задает отношение эффективностей $\eta=\eta_{\mu \mathrm{CE}} / \eta_{\text {МсЕ }}$ мультикалорического и магнитокалорического эффектов (рис. 6). Из него следует, что использование $\mu \mathrm{CE}$ в цикле может как увеличивать, так и уменьшать $\eta$. Причем область увеличения $\eta$ содержит малый интервал от 312 до $314 \mathrm{~K}$, а сам $\eta$ увеличивается не более, чем на $2.5 \%$ для $\mathrm{FeRh}-\mathrm{PZT}$ и $0.5 \%$ для $\mathrm{Cr}_{2} \mathrm{O}_{3}$. Вызвано это тем обстоятельством, что рассматриваемые эффекты являются малыми. Изменение температуры при $\mu \mathrm{CE}$ и МСЕ не превосходит $0.12 \mathrm{~K}$. Увеличения указанных калорических эффектов можно добиться увеличением прилагаемых электрических и магнитных полей. При увеличении магнитного поля до $2 \mathrm{~T}$ и удвоении электрического поля до $2 \cdot 10^{5} \mathrm{~V} / \mathrm{m}$ следует ожидать величину МСЕ порядка $1.2 \mathrm{~K}$, а максимальное увеличение эффективности в 10\%. Эти оценки не противоречат экспериментальным данным, полученным в [24]. В аналогичном железо-родиевом сплаве $\mathrm{Fe}_{49} \mathrm{Rh}_{51}$ изменение температуры для магнитного поля в $2 \mathrm{~T}$ составило $13 \mathrm{~K}$. Для рассматриваемого композита в качестве верхней оценки МСЕ можно взять половину указанной величины, если принять во внимание равную толщину магнитострикционного и пьезоэлектрического слоев и их примерно одинаковые теплоемкости.

Температурная зависимость МЕ-восприимчивости $\beta$ для многих веществ имеет достаточно выраженный максимум для некоторой температуры $T_{0}$. Для $\mathrm{Cr}_{2} \mathrm{O}_{3}$ этот максимум имеет место при $260 \mathrm{~K}$, а для $\mathrm{FeRh}-$ PZT - при $312 \mathrm{~K}$. Экстремумы $\beta$, как правило, расположены вблизи фазовых переходов (электрических или магнитных). Окрестность $T_{0}$ наиболее интересна для тех приложений, где используется взаимное преобразование энергии электрического и магнитного полей. Но в этой окрестности вклад MECE в $\mu \mathrm{CE}$ мал, и для управле- ния тепловыми полями при одновременном приложении электрического и магнитного полей она непригодна. Наибольший вклад MECE в $\mu \mathrm{CE}$ достигается в окрестности максимума производной по температуре от $\beta$ при $T=T_{1}$. Для $\mathrm{Cr}_{2} \mathrm{O}_{3}$ температура $T_{1}=295 \mathrm{~K}$, а для композита FeRh-PZT производная по температуре имеет два экстремума при 310 и $312 \mathrm{~K}$. В окрестности $T_{1} \mathrm{MCE}$ наиболее сильно зависит от электрического поля, что облегчает управление МСЕ посредством электрического поля. Значит, вблизи этой точки электрическое поле дает наибольший вклад в холодопроизводительность. Вторая производная от $\beta$ в этой точке обращается в 0 , и $\mu$ КЭ оказывается одинаково эффективным с MCE. MECЕ наиболее сильно влияет на эффективность в окрестности экстремумов второй производной от $\beta$ по температуре, и наибольшее изменение эффективности достигается при $T=T_{2}$. Для $\mathrm{Cr}_{2} \mathrm{O}_{3}$ температура $T_{2}=300 \mathrm{~K}$, а для $\mathrm{FeRh}-$ PZT наибольшие изменения эффективности наблюдаются в диапазоне $310-315 \mathrm{~K}$.

В описанных материалах увеличение эффективности $\mu \mathrm{CE}$ происходит за счет взаимодействия электрического и магнитного полей. Аналогичного эффекта можно ожидать в пьезоэлектрических материалах, в которых взаимодействуют упругое и электрическое поле. Действительно, пьезоэлектрокалорический эффект в РZT, обусловленный температурной зависимостью пьезоэлектрических коэффициентов, может достигать несколько градусов [30]. Рассчитанная по приведенной в настоящей работе методике разница в эффективностях $\mu \mathrm{CE}$ и ЕСЕ может составлять до 30\% ([31]), правда, тоже в узком температурном диапазоне от 510 до $520 \mathrm{~K}$.

\section{Заключение}

Термодинамический анализ цикла Брайтона для сэндвича FeRh-PZT показал, что максимальное увеличение эффективности для $\mu$ КЭ по сравнению с МСЕ достигается при значениях температуры вблизи точки перегиба температурной зависимости магнитоэлектрического коэффициента. Применение не одиночного магнитного поля, а одновременное воздействие двух полей позволяет увеличить эффективность на 2.5\%. Если же усилить электрическое и магнитное поля по сравнению с использованными в эксперименте, то увеличение эффективности может дойти до $10 \%$. Сравнительно небольшое увеличение эффективности вызвано малостью магнитоэлектрического взаимодействия. Так как из трех имеющихся полей (электрического, магнитного и упругого) наиболее сильно взаимодействуют электрическое и упругое, то значительно большее увеличение эффективности следует ожидать для пьезоэлектриков, обладающих ЕСЕ. Действительно, результаты расчетов по выведенным в работе формулам показали увеличение эффективности в РZT при использовании $\mu \mathrm{CE}$ на $30 \%$ по сравнению с ЕСЕ. 
Полученные результаты позволяют установить температурные области, в которых электрическое поле оказывает наиболее сильно влияние на магнитное поле, а также на МСЕ и на эффективность охлаждения рассматриваемого композита. Относительный СОР $\eta$ и холодопроизводительность $Q_{c}$ являются взаимодополняющими характеристиками. В настоящей работе произведено сравнение $\eta$ для одиночных калорических эффектов с $\mu$ КЭ. При этом предполагалось, что их холодопроизводительность одинаковая. Для практических приложений важную роль играют обе характеристики $\eta$ и $Q_{c}$. Из результатов работы следует, что существует температурная область, в которой использование $\mu \mathrm{CE}$ одновременно увеличивает холодильный коэффициент и холодопроизводительность. Такой областью является область температур от $T_{1}$ до $T_{2}$.

Увеличение термодинамической эффективности сегнетомагнетиков связано с МЕСЕ, который определяется температурной зависимостью магнитоэлектрической восприимчивости. Следовательно, высокая эффективность будет в материалах с большим значением $\beta$. Так как $\beta$ нелинейно зависит от электрического и магнитного полей, то следует выбирать оптимальные значения $E$ и $H$. Вторым способом увеличения эффективности является использование композитов с близкими температурами фазовых переходов. Кроме того, значительное увеличение $\beta$ следует ожидать в области электромеханического резонанса [32].

Таким образом, слоистые структуры являются весьма перспективным материалом для эффективных твердотельных охладителей. Физические свойства слоев должны быть подобраны так, чтобы коэффициенты, описывающие взаимодействие различных полей (магнитоэлектрические, пьезоэлектрические и пьезомагнитные), имели заметную температурную зависимость. Механизмы увеличения эффективности в таких структурах имеют наглядную физическую природу. Значит, использование не одного калорического эффекта, а нескольких позволяет увеличить эффективность твердотельного охлаждения, что дает основания надеяться на разработку высокоэффективных твердотельных охладителей на $\mu \mathrm{CE}$ в ближайшее время.

Работа выполнена при финансовой поддержке Российского Научного Фонда (проект № 18-19-00512). Экспериментальные данные, используемые для моделирования, получены при поддержке Российского Фонда Фундаментальных Исследований (проект № 18-32-01036 мол_а).

\section{Список литературы}

[1] Кириллин В.А., Сычев В.В., Шейндлин А.Е. Техническая термодинамика. Издательский дом МЭИ, 2016. 427 с.

[2] Пахомов О.В., Карманенко С.Ф., Семенов А.А., Старков А.С., Еськов А.В. // ЖТФ. 2010. Т. 80. Вып. 8. C. 80-85. [Pakhomov O.V., Karmanenko S.F., Semenov A.A., Starkov A.S., Eskov A.V. // Tech. Phys. 2010. Vol. 55. N 8. P. 1155-1160.] DOI: $10.1134 / \mathrm{S} 106378421008013 \mathrm{X}$
[3] Флеров И.Н. // Изв. Санкт-Петербургского гос. ун-та низкотемпературных и пищевых технологий. 2008. № 1. C. 41-63.

[4] Старков А.С., Старков И.А. // ЖЭТФ. 2014. Т. 146. Вып. 2. C. 297-303. [Starkov A.S., Starkov I.A. // J. Exp. Theor. Phys. 2014. Vol. 119. N 2. P. 258-263.] DOI: $10.1134 / \mathrm{S} 1063776114070097$

[5] Suchaneck G., Gerlach G. // Phys. Scr. 2015. Vol. 90. N 9. P. 094020-6. DOI: 10.1088/0031-8949/90/9/094020

[6] Флёров И.Н., Михалёва Е.А., Горев М.В., Карташев А.В. // ФTТ. 2015. Т. 57. Вып. 3. С. 421-431. [Flerov I.N., Mikhaleva E.A., Gorev M.V., Kartashev A.V. Phys. Solidi State. 2015. Vol. 57. N 429. P. 429-441.] DOI: $10.1134 / \mathrm{S} 1063783415030075$

[7] Tishin A.M., Spichkin Y.I. The magnetocaloric effect and its applications. Bristol, Philadelphia: Institute of Physics Publishing, 2003. 475 p.

[8] Cui J., Wu Y., Muehlbauer J., Hwang Y., Radermacher R., Fackler S., Wuttig I., Takeuchi I. // Appl. Phys. Lett. 2012. Vol. 101. N 7. P. 073904. DOI: $10.1063 / 1.4746257$

[9] Guo D., Gao J., Yu Y.J., Santhanam S., Slippey A., Fedder G.K., McGaughey A.J.H., Yao S.-C. // Int. J. Heat Mass Transfer. 2014. Vol. 72. P. 559-564. DOI: 10.1016/j.ijheatmasstransfer.2014.01.043

[10] Sinyavsky Y.V., Pashkov N.D., Gorovoy Y.M., Lugansky G.E., Shebanov L. // Ferroelectrics. 1989. Vol. 90. N 1. P. 213-217. DOI: $10.1080 / 00150198908211296$

[11] Zimm C., Jastrab A., Sternberg A., Pecharsky V., Gschneidner K. Jr., Osborne M., Anderson I. Advances in cryogenic engineering. Springer US, 1998. P. 1759-1766.

[12] Старков А.С., Пахомов О.В., Старков И.А. // Письма в ЖТФ. 2011. Т. 37. Вып. 23. С. 105-110. [Starkov A.S., Pakhomov O.V., Starkov I.A. // Techn. Phys. Lett. 2011. Vol. 37. N 12. P. 1139-1141.] DOI: $10.1134 / \mathrm{S} 1063785011120133$

[13] Fahler S., Rossler U.K., Kastner O., Eckert J., Eggeler G., Emmerich H., Entel P., Muller S., Quandt E., Albe K. /I Adv. Eng. Mater. 2012. Vol. 14. N 1-2. P. 10-19. DOI: 10.1002/adem. 201100178

[14] Starkov I.A., Starkov A.S. // IEEE T. Ultrason. Ferr. 2014. Vol. 61. N 8. P. 1357-1363. DOI: 10.1109/TUFFC.2014.3043

[15] Starkov I.A., Starkov A.S. // arXiv preprint. 2015. arXiv:1602.04238

[16] Starkov I.A., Starkov A.S. // Int. J. Solid. Struct. 2016. Vol. 100. P. 187-194. DOI: 10.1016/j.jijsolstr.2016.08.015

[17] Смоленский Г.А., Чупис И.Е. // УФН. 1982. Т. 137. Вып. 7. C. 415-448. [Smolenskii G.A., Chupis I.E. // Sov. Phys. Uspekhi. 1982. Vol. 25. N 7. P. 475-508.]

[18] Пятаков А.П., Звездин А.К. // УФН. 2012. Т. 182. Вып. 6. C. 593-620. [Pyatakov A.P., Zvezdin A.K. // Phys. Usp. 2012. Vol. 55. N 6. P. 557-584.] DOI: $10.3367 /$ UFNr.0182.201206b.0593

[19] Ландау Л.Д., Лившии, Е.М. Электродинамика сплошных сред. М.: Физматлит, 1992. 664 с.

[20] Старков А.С., Пахомов О.В., Старков И.А. // Письма в ЖЭТФ. 2010. Т. 91. Вып. 10. С. 556-560. [Starkov A.S., Pakhomov O.V., Starkov I.A. // JETP Lett. 2010. Vol. 91. N 10. P. 507-511. DOI: $10.1134 / \mathrm{S} 002136401$

[21] Kitanovski A., Egolf P.W. // IJR. 2006. Vol. 29. N 1. P. 3-21. DOI: $10.1016 /$ j.ijrefrig.2005.04.007

[22] Aliev A.M., Batdalov A.B., Khanov L.N., Kamantsev A.P., Koledov V.V., Mashirov A.V., Shavrov V.G., Grechishkin R.M., Kaul' A.R., Sampath V. // Appl. Phys. Lett. 2016. Vol. 109. P. 202407(1)-220407(5). DOI: 10.1063/1.4968241 
[23] Zakharov A.I., Kadomtseva A.M., Levitin R.Z., Ponyatovskii E.G. // Sov. Phys. JETP-USSR. 1964. Vol. 19. P. $1348-1353$.

[24] Nikitin S., Myalikgulyev G., Tishin A., Annaorazov M., Asatryan K., Tyurin A. // Phys. Lett. A. 1990. Vol. 148. P. 363-366. DOI: $10.1016 / 0375-9601(90) 90819-A$

[25] Aliev A.M. // arXiv preprint. 2014. arXiv:1409.6898.25.

[26] Aliev A.M., Batdalov A.B., Khanov L.N., Koledov V.V., Shavrov V.G., Tereshina I.S., Taskaev S.V. // J. Alloys Compd. 2016. Vol. 676. P. 601-605.

DOI: $10.1016 /$ j.jallcom.2016.03.238

[27] Gridnev S.A., Kalgin A.V., Amirov A.A., Kamilov I.K. // Ferroelectrics. 2010. Vol. 397. N 1. P. 142-150. DOI: $10.1080 / 00150193.2010 .484775$

[28] Астров Д.Н. // ЖЭТФ. 1961. Т. 40. № 4. С. 1035-1041. [Astrov D.N. // Sov. Phys. JETP-USSR. 1961. Vol. 13. N 4. P. 729-734.]

[29] Garcia V., Fusil1 S., Briddon P.R., Guiblin N., Mougin A., Ünal A.A., Kronast F., Valencia S., Dkhil B., Barthélémy A., Bibes M. // Nature Mater. 2014. Vol. 13. N 4. P. 345-351. DOI: $10.1038 / \mathrm{NMAT} 3870$

[30] Старков А.С., Старков И.А. // ФТТ. 2016. Т. 58. Вып. 9. C. 1739-1743. [Starkov A.S., Starkov I.A. // Phys. Solid State. 2016. Vol. 58. N 9. P. 1798-1803.] DOI: $10.1134 / \mathrm{S} 1063783416090298$

[31] Старков А.С., Пахомов О.В., Родионов В.В., Амиров А.А., Старков И.А. // Письма в ЖТФ. 2018. Т. 44. Вып. 6. С. 4248.

[32] Филиппов Д.А., Бичурин М.И., Петров В.М., Лалетин В.М., Поддубная H.H., Srinivasan G. // Письма в ЖТФ. 2004. Т. 30. Вып. 1. С. 15-20. [Filippov D.A., Bichurin M.I., Petrov V.M., Laletin V.M., Poddubnaya N.N., Srinivasan G. // Techn. Phys. Lett. 2004. Vol. 30. N 1. P. 6-8.] DOI: $10.1134 / 1.1646700$ 